\title{
REMYP_07 Evaluación de la adquisición de la Competencia Transversal "Responsabilidad Ética, Medioambiental y Profesional" en los estudios de grado de la Universitat Politècnica de València.
}

Ignacio Andrés-Doménecha, Marta Cabedo Fabres ${ }^{\mathrm{a}}$, Antonio Martí-Campoya , Eloïna Coll Aliaga ${ }^{\mathrm{a}}$, Ernesto Fenollosa Fornera, Ester Gimenez-Carbo a, M. Esther Gómez-Martín ${ }^{a}$, J. Félix Lozano Aguilar $^{\mathrm{a}}$, Alicia Llorca Ponce ${ }^{\mathrm{a}}$, Núria Pascual-Seva ${ }^{\mathrm{a}}$, M. Teresa Sebastiá Frasquet ${ }^{\mathrm{a}}$, Maria Vargas ${ }^{\mathrm{a}}$. anniversitat Politècnica de València, esgimen@cst.upv.

\section{\$EWWDFW}

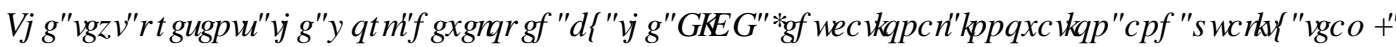

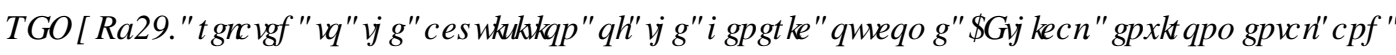

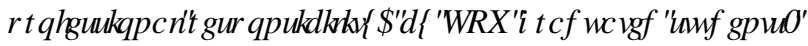

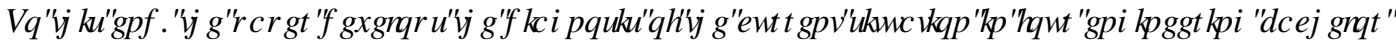

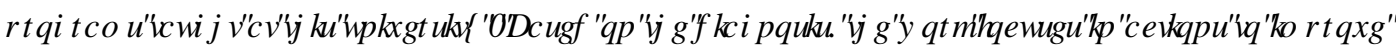

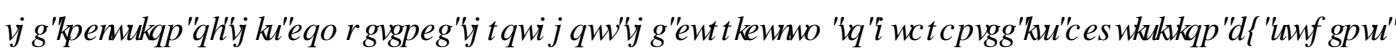
ZKRIFRP SOAMIKKHUWXGHW

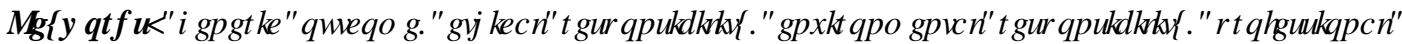
UHSRQMEICWMHDDODMRQI

\section{HXP HQ]}

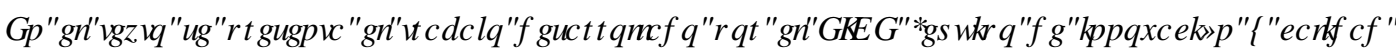

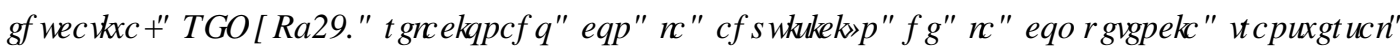

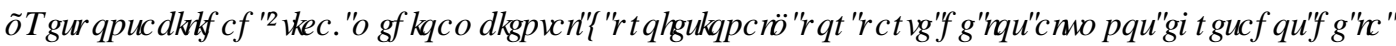
$839 \square$

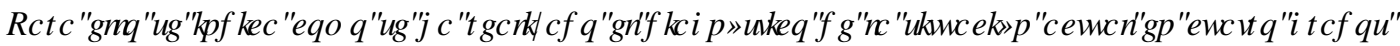

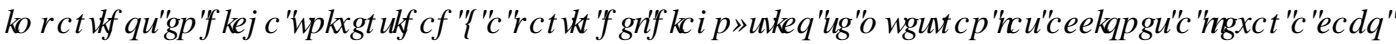

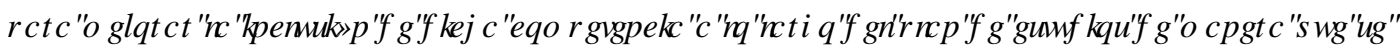

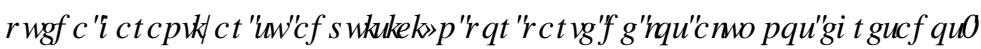

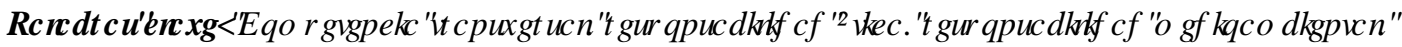
UHSRQMEICOCOFSURIHMRQDOHIDODFIYQ

\section{Introducción}

El proyecto de competencias transversales (CT) de la Universitat Politècnica de València (UPV) comenzó su andadura en el año 2013 y tiene como objetivo principal acreditar que los alumnos egresados en cualquiera de las titulaciones impartidas en la Universitat Politècnica de València las ha adquirido. Hacia finales del curso 2013-14 se impartieron actividades formativas en todos los centros presentando el proyecto y trasladando a los equipos directivos de las diferentes Escuelas la necesidad de comenzar a realizar actividades piloto durante el curso 2014-15 con el objetivo de que en el curso académico 2015/16 se produjese la implantación definitiva del proyecto. 
En las 13 Escuelas de la UPV, se establecieron diferentes asignaturas como puntos de control de cada uno de los niveles de dominio de las trece competencias transversales establecidas por el Vicerrectorado de Estudios, Calidad y Acreditación. Para cada competencia se establecieron dos niveles de dominio correspondientes a los estudios de grado, y un tercer nivel de dominio que corresponde a los estudios de máster.

En el curso escolar 2017-18, empezaron a titularse alumnos de grado y de máster que habían completado todos los niveles de dominio correspondientes a sus estudios y por ello estamos ahora en situación de comprobar el estado de adquisición de las distintas competencias transversales.

En este contexto se creó el Equipo de Innovación y Calidad Educativa (EICE) REMYP_07, con la finalidad de comprobar el estado de adquisión de la competencia transversal "Responsabilidad ética, medioambiental y profesional". Este equipo de trabajo inició en el curso 2020-21 el Proyecto de Inmovación y Mejora Educativa (PIME) titulado "Evaluación del nivel de adquisición de la CT07 Responsabilidad ética, medioambiental y profesional en los estudios de grado de la UPV. Propuestas de mejora". El trabajo se iniciará con el estudio de los dos niveles de dominio establecidos para los distintos grados y en los años posteriores seguirá con el trabajo desarrollado afrontando los estudios de máster.

Los niveles de dominio que deben alcanzar los estudiantes al completar los estudios de grado en esta competencia transversal se concretan en los resultados de aprendizaje e indicadores que se muestran en las tablas 1 y 2. Cabe destacar que es la única competencia transversal en que se trabaja en dos dimensiones: por una parte la responsabilidad ética y profesional (Tabla 1) y, por otra, la responsabilidad medioambiental (Tabla 2) lo que hace más complejo su adquisición.

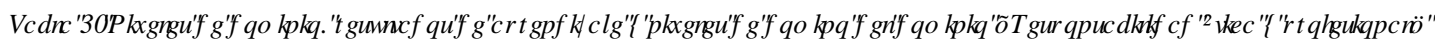

\begin{tabular}{cl}
$\begin{array}{c}\text { Nivel de } \\
\text { Dominio }\end{array}$ & \multicolumn{1}{c}{ Resultados de aprendizaje } \\
\hline 1 & $\begin{array}{l}\text { Cuestionar la realidad y ser conscientes de los conceptos y valores a partir de los que } \\
\text { se construye la misma. }\end{array}$ \\
2 & $\begin{array}{l}\text { Analizar críticamente los juicios propios y ajenos sobre la realidad, y ser conscientes } \\
\text { de las consecuencias e implicaciones de estos. }\end{array}$ \\
3 & $\begin{array}{l}\text { Mostrar y argumentar la pertinencia de los comportamientos y juicios que se emiten, } \\
\text { fundamentados con conceptos éticos y deontológicos. }\end{array}$
\end{tabular}

Fuente: UPV (2021)

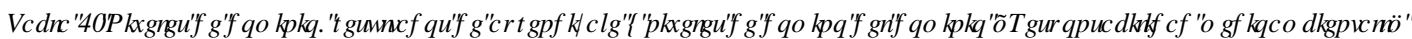

\begin{tabular}{cl}
\hline $\begin{array}{c}\text { Nivel de } \\
\text { Dominio }\end{array}$ & \multicolumn{1}{c}{ Resultados de aprendizaje } \\
\hline 1 & $\begin{array}{l}\text { Entender la necesidad de valorar las consecuencias de las actuaciones profesionales } \\
\text { en cuanto a repercusión social, ambiental y económica, y actuar consecuentemente. }\end{array}$ \\
2 & $\begin{array}{l}\text { Diseñar, organizar y aplicar actuaciones profesionales específicas, respetuosas con } \\
\text { el entorno social, económico y ambiental. }\end{array}$ \\
3 & $\begin{array}{l}\text { Coordinar y evaluar actuaciones integrales en el ámbito profesional respetuosas con } \\
\text { el entorno social, económico y ambiental. }\end{array}$
\end{tabular}

Fuente: UPV (2021) 
Parte de los integrantes del Equipo REMYP_07 pertenecían al grupo de trabajo creado por el Instituto de Ciencias de la Educación encargado de diseñar las rúbricas institucionales para la evaluación de la CT-07. A este núcleo inicial se han unido otros docentes con un manifiesto interés en la formación de los egresados de la UPV.

\section{Objetivos}

El objetivo general del EICE REMYT_07 es estudiar y realizar un diagnóstico del estado de adquisición de la competencia transversal CT-07 "Responsabilidad ética, medioambiental y profesional", en cada uno de los niveles de dominio programados en los estudios de grado y máster impartidos en la UPV.

Este diagnóstico incluye la revisión de las metodologías, actividades, evidencias y rúbricas utilizadas, así como el estudio de la idoneidad de las asignaturas seleccionadas como punto de control.

A partir de este diagnóstico, se estudiarán acciones de mejora que pueden abarcar desde la mejora de las rúbricas institucionales para adaptarlas al contexto, hasta el desarrollo de acciones formativas coherentes con la CT-07.

Este objetivo general se concretará con los siguientes objetivos específicos:

OE1. Diagnosticar el grado de adquisición de la competencia en la actualidad. En esta fase del proyecto, se analizarán las guías docentes de las asignaturas punto de control para la adquisición de la CT 07 en el plan de estudios de cada uno de los grados.

OE2. Analizar el plan de estudios de cada titulación y seleccionar las asignaturas más adecuadas para introducir la competencia transversal. En esta fase del proyecto se estudiarán las asignaturas más adecuadas del plan de estudios para incorporar la CT07, y asegurar que al completar los estudios los alumnos han adquirido el nivel de dominio requerido para cada nivel de dominio de la competencia.

OE3. Introducir cambios a nivel de asignaturas y/o materias para la incorporación de la competencia transversal, tanto a nivel de contenidos como de herramientas para su evaluación. En esta fase del proyecto se concebirán y definirán las actividades formativas transversales y obligatorias para los estudiantes de distintos cursos, adecuadas al nivel de dominio requerido. Se incorporarán nuevas herramientas de evaluación, para asegurar la adquisición de la competencia, así como la adecuada recogida de evidencias y la correcta calificación. Este nivel se corresponde con propuestas de mejora que no suponen una modificación de la memoria de verificación del título.

OE4. Seguimiento de la evolución del alumno y de la adquisición de los dos niveles de dominio requeridos en el grado. Tras la revisión e implementación de nuevas herramientas para la incorporación de la CT07 en distintas asignaturas específicas de los grados, se estudiará la evolución y el grado de adquisición de la competencia por parte de los alumnos, en los niveles de dominio requeridos.

OE5. Plantear posibles mejoras del plan de estudios, que puedan dar lugar a cambios más integrados y articulados respecto de la adquisición de la CT-07 "Responsabilidad ética, medioambiental y profesional" por parte de todos los egresados de la UPV. El tercer nivel se corresponde con propuestas de mejora que suponen una modificación de la memoria de verificación del título. El margen de acción y la inmediatez en la implantación de estas propuestas es más limitado, pero conviene estudiar su necesidad y viabilidad. 
Todo ello para conseguir que los alumnos egresados adquieran y evidencien la adquisición de la CT-07. En este trabajo se presentan los resultados de completar el objetivo específico OE1.

\section{Desarrollo de la innovación}

\subsection{Metodología}

En el inicio de la formación del EICE, se invitó a docentes de las once escuelas y dos facultades de la UPV a participar en él. Una vez constituido y en función de los integrantes, se seleccionaron los grados en ingeniería en los que se empezó a trabajar. Se elaboró un plan de trabajo, nombrando responsables de cada una de las tareas para facilitar y asegurar el cumplimiento de las mismas.

Desde el inicio del curso 2019-2020, se han tenido periódicamente reuniones de coordinación, a las que asisten al menos el responsable y los docentes encargados de cada tarea y se invita al resto de miembros a participar. En estas reuniones se pone en común el trabajo desarrollado, se discute el modo en que abordar las tareas, se elaboran resúmenes y todo el material que se va produciendo se comparte en un espacio reservado de la plataforma Poliformat a la que tienen acceso los componentes de REMYP-07.

También en estas reuniones de coordinación se ha elaborado una dinámica grupal para realizar con el alumnado del último curso del grado y se ha elaborado una guía para realizar entrevistas a los docentes implicados.

El método de trabajo elegido está resultando eficaz, así que para el avance de las siguientes tareas se continuará elaborando y analizando material de manera individual y posteriormente se realizaran reuniones grupales en las que se coordinará el trabajo desarrollado.

\subsection{Plan de trabajo}

Para desarrollar la innovación se ha elaborado un plan de trabajo inicial. Para ello se han seleccionado las titulaciones en las que se revisará e implementarán mejoras. Posteriormente y tras validar el sistema de trabajo se extenderá la tarea a todas las titulaciones impartidas en la UPV. El plan de trabajo a largo plazo abarcará el estudio del estado de adquisición de la CT-07 por todos los egresados de los 13 centros de la UPV.

Para acometer esta tarea, se va a iniciar el trabajo abordando el análisis de cuatro grados de ingeniería impartidos en cuatro Escuelas de la UPV, y que son; Grado en Ingeniería de Tecnologías y Servicios de Telecomunicación en la ETSIT, Grado en Ingeniería Agroalimentaria y del Medio Rural en la ETSIAMN, Grado en Ingeniería Civil en la ETSICCP y Grado en Ingeniería Geomática y Topografía en la ETSIGCT.

En el curso 2020-21, con el inicio del PIME "Evaluación del nivel de adquisición de la CT07 Responsabilidad ética, medioambiental y profesional en los estudios de grado de la UPV. Propuestas de mejora", se han incorporado al análisis el Grado en Fundamentos de Arquitectura en la ETSA y el Grado en Ciencias Ambientales de la EPSG.

Como ya se ha dicho, en cursos sucesivos se ampliará el número de grados, de másteres y de escuelas analizadas. Se pretende que el EICE sea dinámico y estará abierto a incorporar al equipo de trabajo a cualquier docente que manifieste su deseo de colaborar en el proyecto.

En cada una de las Escuelas, para cada grado seleccionado para desarrollar el estudio, se realizarán las siguientes tareas, vinculadas con cada uno de los objetivos específicos:

Tarea 1: Revisión de las asignaturas que en la actualidad son punto de control de la CT-07, y recopilación de las evidencias de adquisición de la competencia. 
Tarea 2: Análisis de las evidencias y adecuación a los niveles de dominio de la adquisición de la competencia.

Tarea 3: Selección de las asignaturas del plan de estudios más adecuadas para ser punto de control de la CT-07.

Tarea 4: Diseño de actividades y adecuación de rúbricas de las asignaturas punto de control.

Tarea 5: Utilización de las nuevas actividades y rúbricas en las asignaturas punto de control "piloto".

Tarea 6: Recopilación de las evidencias de adquisición de la competencia y análisis de la evaluación.

Tarea 7: Seguimiento de la evolución del alumnado y de la adquisición de los dos niveles de dominio requeridos en el grado y el tercer nivel de dominio requerido en el máster.

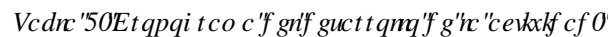

\begin{tabular}{|c|l|l|l|l|l|l|l|l|}
\hline & \multicolumn{3}{|c|}{ CURSO 2020-2021 } & \multicolumn{4}{c|}{ CURSO 2021-2022 } \\
\hline Tarea 1 & & & & & & & & \\
\hline Tarea 2 & & & & & & & & \\
\hline Tarea 3 & & & & & & & & \\
\hline Tarea 4 & & & & & & & \\
\hline Tarea 5 & & & & & & & & \\
\hline Tarea 6 & & & & & & & \\
\hline Tarea 7 & & & & & & & & \\
\hline
\end{tabular}

) XHQUALC (DERLFIYQSLRSIDI

\section{Resultados}

En estos momentos se ha completado totalmente la tarea 1 y se va a finalizar la tarea 2 . Una de las particularidades de la competencia transversal "Responsabilidad ética, medioambiental y profesional"(CT07), es que es la única que posee dos rúbricas para cada nivel de dominio, ya que es diferente evaluar la responsabilidad ética y profesional o la responsabilidad medioambiental. Esto añade dificultad en el momento de evaluar si los egresados han adquirido la competencia, ya que se puede dar el caso de que todas las asignaturas punto de control del plan de estudios sólo evalúen uno de estos dos aspectos.

Los resultados obtenidos al completar la Tarea 1: "Revisión de las asignaturas que en la actualidad son punto de control de la CT-07, y recopilación de las evidencias de adquisición de la competencia.”, se pueden resumir del siguiente modo:

- Todas las asignaturas de las que tenemos evidencias, adecúan la dificultad de la actividad relacionada con la CT a la dificultad del curso.

- Ninguna asignatura utiliza las rúbricas institucionales para realizar la evaluación.

- Entre los métodos para evaluar presentados hay rúbricas simplificadas y cuestionarios con escala de Likert.

- Excepto dos asignaturas de todos los grados analizados, solo se trabaja uno de los dos aspectos de la competencia (o el ambiental o la responsabilidad profesional y ética). Esto va a provocar que los alumnos no adquieran alguno de los aspectos de la CT-07.

- La mayoría de las actividades están contextualizadas en la asignatura específica. 
- En aproximadamente la mitad de las asignaturas analizadas se presentan evidencias relacionadas con lo que dice la guía docente sobre la CT, pero en la otra mitad hay diferencias notables entre lo que se hace en clase y lo que esta previsto en la guía docente.

- No es evidente que con las actividades propuestas en cada asignatura se alcance el nivel de dominio deseado de la competencia transversal.

Tras completar la primera tarea se pasó a la siguiente actividad que según el plan de trabajo consiste en el "análisis de las evidencias y adecuación a los niveles de dominio de la adquisición de la competencia."

Para poder acometer esta tarea con la máxima información posible, se planteó la realización de una dinámica grupal con alumnos de último curso de los estudios de grado (Figura 1), así como entrevistas personales a los docentes responsables de las asignaturas punto de control para conocer su visión sobre el programa institucional en general y de cómo se adquiere la CT-07 por parte de los alumnos en particular. Además, nos servimos de estos escenarios para empezar a pre-"seleccionar las asignaturas del plan de estudios más adecuadas para ser punto de control de la CT-07” (tarea 3). Entre los resultados obtenidos de los estudiantes se pueden destacar los siguientes:

En relación con el programa institucional, de manera general, la impresión es que los alumnos conocen poco del proyecto. Saben que existen competencias transversales, muchos no conocían las 13 competencias ni los niveles de dominio de cada competencia y recuerdan algunas asi gnaturas en que se ha evaluado alguna competencia transversal, pero no muy claramente.
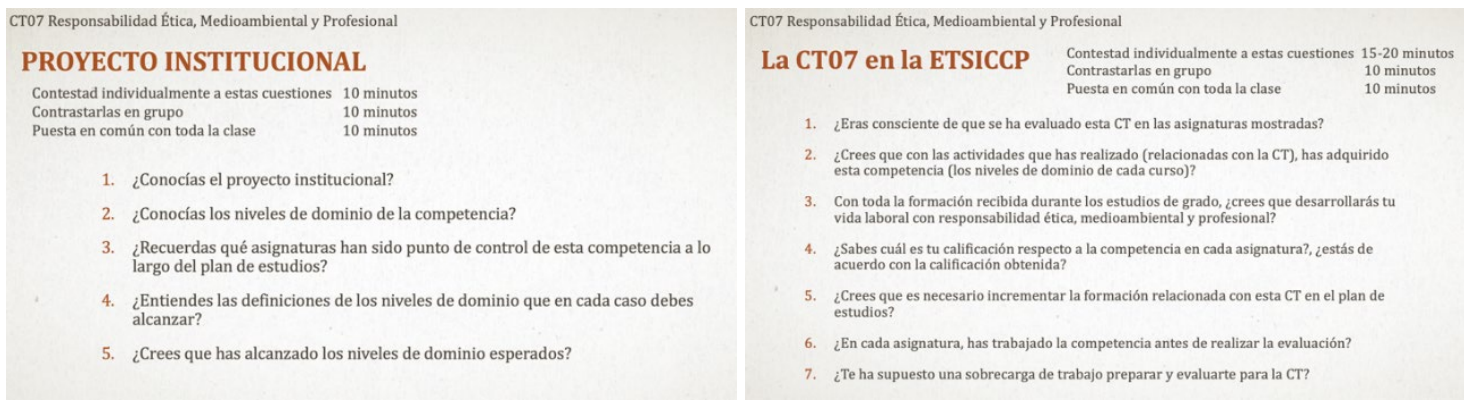

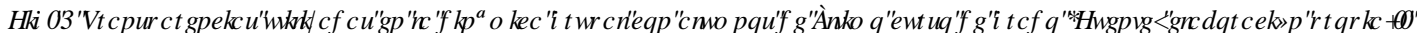

Y en relación con su percepción sobre si han adquirido la CT-07 en los niveles de dominio deseados se muestran bastante disconformes. Entre las críticas que expresan se pueden destacar las siguientes. En algunas de las asignaturas punto de control no recuerdan haber hecho ninguna actividad relacionada con la CT-07, piensan que las actividades no están bien relacionadas con el nivel de dominio a adquirir y muestran disconformidad con el hecho de que se les evalúe una competencia para la que no han recibido formación. Se muestran descontentos por ser, en algunos casos, evaluados de una CT, en este caso responsabilidad ética medioambiental y profesional, de la que no son conscientes de que se trabaja y evalúa.

En cuanto a las entrevistas a los docentes se están realizando en estos momentos, y a partir del análisis de los resultados se continuará con la tarea 3 y 4 (Tarea 3: Selección de las asignaturas del plan de estudios más adecuadas para ser punto de control de la CT-07; Tarea 4: Diseño de actividades y adecuación de rúbricas de las asignaturas punto de control).

\section{Conclusiones}

Como queda plasmado en el texto, el trabajo no está finalizado, pero tras meses de trabajo ya se pueden extraer las primeras conclusiones. El objetivo del equipo REMYP_07 es trabajar con la CT_07, pero no se 
puede olvidar que esta competencia transversal es una de las trece que se pretende que adquieran todos los egresados de la UPV. La consecución del apoyo y financiación a través del PIME “ Evaluación del nivel de adquisición de la CT07 Responsabilidad ética, medioambiental y profesional en los estudios de grado de la UPV. Propuestas de mejora“ ha resultado un impulso para seguir esta línea de trabajo. Por ello se puede decir:

- En estos momentos es necesario evaluar el estado de desarrollo en que se encuentra el proyecto institucional de incorporar 13 competencias transversales en todas las titulaciones impartidas en la Universitat Politècnica de Valencia.

- Este análisis es necesario para no transmitir a los docentes la sensación de que "ya ha pasado el tiempo de las competencias transversales".

- Aunque el trabajo presentado únicamente va a evaluar el estado en que se encuentra la CT "responsabilidad ética, medioambiental y profesional", se han recogido muchas opiniones relacionadas con el número de competencias transversales que adquieren nuestros estudiantes. Si vamos a certificar su adquisición debemos estar seguros que las adquieren. Además, conviene abrir el debate sobre el número de competencias transversales que considera la UPV, en línea con otras instituciones académicas, como por ejemplo ABET (Accreditation Board for Engineering and Technology), que las está reduciendo (en estos momentos, siete).

- De manera general se puede decir que no se han recogido las suficientes evidencias para poder certificar que los alumnos de las cuatro titulaciones analizadas han adquirido la CT para el nivel de dominio requerido, al finalizar sus estudios grado.

- Es necesario tratar la CT-07 como dos competencias transversales, ya que si únicamente evaluamos alguno de los aspectos (el medioambiental o la responsabilidad ética y profesional), no se puede garantizar que la competencia se ha adquirido completamente.

- Las rúbricas institucionales para evaluar la CT pueden resultar demasiado complejas e incluso confusas. Esto puede haber contribuido a que en las asignaturas analizadas hasta el momento no se utilicen.

- Es necesario explicar correctamente a los alumnos la finalidad de las CTs, nombrar las que se trabajan en cada una de las asignaturas específicas (especialmente si se van a evaluar), y dar formación relacionada con la competencia antes de valorar si los estudiantes han alcanzado el nivel de dominio deseado y en que medida (que calificación obtienen). Los alumnos quieren aprender.

- Para que el proyecto tenga éxito es imprescindible la colaboración del profesorado responsable de las asignaturas punto de control. Esta competencia transversal es difícil de trabajar y de evaluar, por ello se debe proporcionar toda la ayuda y colaboración posible a los docentes implicados.

Desde el equipo REMYP_07 estamos trabajando para conocer si nuestros alumnos reciben formación y son evaluados adecuadamente para acreditar que adquieren la competencia "responsabilidad ética, medioambiental y profesional". Pensamos que esta es una competencia imprescindible para poder desarrollar cualquier profesión de manera excelente. Esta opinión está respaldada por el hecho de que agencias internacionales que acreditan la calidad de diferentes estudios universitarios como ABET, tienen entre los siete resultados de aprendizaje que los alumnos deben tener al terminar sus estudios universitarios

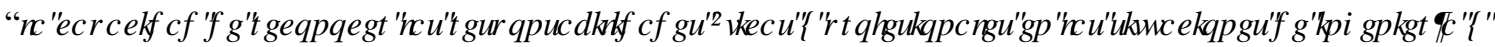

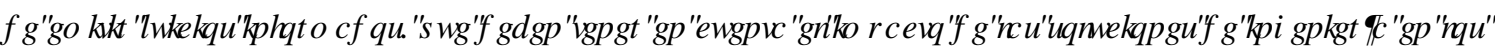
FRQUA URKP XQADOHFRQ P IFRIDP EIHQWDO IVFIDO. 


\section{Referencias}

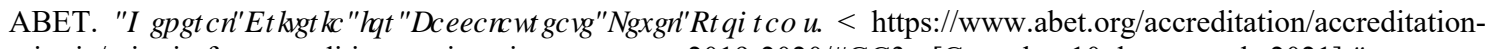
criteria/criteria-for-accrediting-engineering-programs-2019-2020/\#GC3> [Consulta: 10 de marzo de 2021] ]

UNIVERSITAT POLITÈCNICA DE VALÈNCIA. \&RPSHHQFWV WDQMHUDON $<$ http://www.upv.es/contenidos/COMPTRAN/info/955712normalc.html> [Consulta: 10 de marzo de 2021]

UNIVERSITAT POLITĖCNICA DE VALÈNCIA. \&RPSHWQFWW WDQMHWON. $<$ http://www.upv.es/entidades/ICE/info/Proyecto_Institucional_CT.pdf $>$ [Consulta: 10 de marzo de 2021] 